\title{
Trendelenburg-Related Brachial Plexus Injuries in Gynecologic Surgery
}

\author{
Nigel Pereira, MD, Loretta Hallock, DO, Colleen Yen, BS, Irene Grias, DO, Minda A. Green, MD \\ Department of Obstetrics and Gynecology, Drexel University College of Medicine, Philadelphia, PA, USA (Dr. Pereira). \\ Department of Surgery, Danbury Hospital, Danbury, CT, USA (Dr. Hallock). \\ Drexel University College of Medicine, Philadelphia, PA, USA (Dr. Yen). \\ Division of Minimally Invasive Surgery, Department of Obstetrics and Gynecology, Drexel University College of Medicine, \\ Philadelphia, PA, USA (Drs. Grias, Green).
}

\begin{abstract}
Introduction: Brachial plexus injuries are infrequent but preventable complications of laparoscopic and robot-assisted gynecologic surgery.

Case Description: A 32-year-old woman with a history of uterine leiomyomata, menorrhagia, and chronic pelvic pain underwent a robot-assisted laparoscopic myomectomy. Preoperative radiologic imaging showed an enlarged uterus with a large, $8.6 \times 7.2 \times 9.2-\mathrm{cm}$, intramural left uterine body leiomyoma. Bleeding and difficulty visualizing the surgical dissection planes complicated intraoperative enucleation of the leiomyoma. This resulted in a total surgical time of 400 minutes, during which the patient spent approximately 320 minutes in the steep Trendelenburg position. On postoperative day 1 , the patient reported weakness and tingling in her left arm and fingers and was found to have an acute left brachial plexus injury. After a course of oral corticosteroids and outpatient physical therapy, the patient reported no residual neurologic deficits during her subsequent postoperative visits.

Discussion: As gynecologists cope with the learning curve associated with laparoscopic and robot-assisted laparoscopic surgery, longer operating times will be encountered, with patients spending a significant amount of intraoperative time in the Trendelenburg position. The resulting risks of intraoperative nerve injuries, particularly brachial plexus injuries, may therefore be higher than expected. Because these injuries can cause significant postoperative morbidity, and sometimes even have medicolegal implications, every effort should be made to prevent them. To achieve this, we emphasize the combined efforts of the nursing, surgical, and anesthesia teams to ensure proper patient positioning in the operating room.
\end{abstract}

Key Words: Brachial plexus injury, Laparoscopy, Robotics, Patient positioning, Trendelenburg.

Citation Pereira N, Hallock L, Yen C, Grias I, Green MA. Trendelenburg-related brachial plexus injuries in gynecologic surgery. CRSLS e2014.00077. DOI 10.4293/CRSLS.2014.00077.

Copyright $\odot 2014$ SLS This is an open-access article distributed under the terms of the Creative Commons Attribution-Noncommercial-ShareAlike 3.0 Unported license, which permits unrestricted noncommercial use, distribution, and reproduction in any medium, provided the original author and source are credited.

All authors have nothing to disclose.

The authors acknowledge Diana Winters, BA (Academic Publishing Services), for manuscript preparation.

Address correspondence to: Nigel Pereira, MD, Department of Obstetrics and Gynecology, Drexel University College of Medicine, 245 N 15th St, MS 495, 16th Floor, New College Bldg, Philadelphia, PA 19102, USA. Telephone: (215) 762-8292, Fax: (215) 762-1470, E-mail: Nigel.Pereira@drexelmed.edu

\section{INTRODUCTION}

Gynecologic surgery has undergone a dramatic transformation in the past decade because of refinement of laparoscopic techniques. Although the laparoscopic approach offers smaller incisions, decreased blood loss, and shorter hospitalizations compared with traditional laparotomy, nerve injuries due to patient positioning may occasionally complicate laparoscopic surgery. ${ }^{1-3}$ Brachial plexus injuries (BPIs) are uncommon nerve injuries that may occur during laparoscopic and robot-assisted surgery. ${ }^{1,2}$ We present the case of a patient in whom a BPI developed after a lengthy robot-assisted laparoscopic myomectomy. In addition to reviewing the risks that predispose patients 
to this complication, we provide tips and techniques to help circumvent this infrequent complication.

\section{CASE}

A 32-year-old white woman, gravida 0 , with a history of uterine leiomyomata, menorrhagia, and chronic pelvic pain presented for counseling regarding definitive surgical therapy. Various medical modalities had previously failed, including tranexamic acid and a levonorgestrel intrauterine device. The patient's medical and surgical history was unremarkable. Preoperative transvaginal and transabdominal ultrasonography showed an enlarged uterus measuring $11.4 \times 9.9 \times 9.9 \mathrm{~cm}$ with an intramural left uterine body leiomyoma measuring $8.6 \times 7.2 \times 9.2 \mathrm{~cm}$. Minimally invasive surgical options were discussed with the patient, and with the aim of fertility preservation, the patient opted for a robotassisted laparoscopic myomectomy.

The patient was placed in a dorsal lithotomy position for the procedure. Her arms and hands were padded with 2-inch-thick egg crate foam cushions (Universal Medical, Norwood, Massachusetts), and using the sheet under her back, we tucked both her arms straight to her sides with her palms open and facing her thighs. The patient lay directly on top of a 4-inch-thick egg crate foam mattress (BioClinic; Joerns Medical, Charlotte, North Carolina), and the patient's back sheet was tucked under this foam mattress. In anticipation of steep Trendelenburg positioning during surgery, we attached padded shoulder braces (Allen Medical Systems, Acton, Massachusetts) to the operating table. The patient's position on the operating table was confirmed at a $0^{\circ}$ and $30^{\circ}$ tilt by the surgical, anesthesia, and scrub teams.

After placement of a $12-\mathrm{mm}$ supraumbilical port, two 8-mm ports, and an additional $10-\mathrm{mm}$ accessory port, the standard da Vinci surgical system (Intuitive Surgical, Sunnyvale, California) was straight docked between the patient's legs. The leiomyoma was identified intraoperatively, and dilute vasopressin solution was injected around the leiomyoma site with a 22-gauge spinal needle until blanching of the myometrium was noted. A longitudinal hysterotomy was created, the leiomyoma capsule was identified, and the leiomyoma was enucleated with an EndoWrist tenaculum (Intuitive Surgical) and a monopolar cutting device. Intraoperative enucleation of the leiomyoma was made challenging by the presence of adenomyosis, which made visualization of the surgical planes difficult. This step also resulted in greater-than-usual intraoperative bleeding, primarily due to traction applied to the leiomyoma. The hysterotomy was closed in 3 layers with a No. 2-0 V-Loc suture (Covidien, Mansfield, Massachusetts), and the leiomyoma was extracted from the abdomen by use of a morcellator. The estimated blood loss was $300 \mathrm{~mL}$, and the total surgical time was 400 minutes; the patient spent approximately 320 minutes in the steep Trendelenburg position.

On postoperative day 1 , the patient reported weakness and tingling in her left arm and fingers. A general physical examination showed erythema and tenderness of the left shoulder. A detailed neurologic examination was significant for $3 / 5$ motor strength of the left biceps and wrist extensors, as well as +1 biceps and triceps reflexes. Touch and temperature sensation remained normal throughout the left arm. Magnetic resonance imaging of the cervical spine showed negative findings for cervical vertebral fracture, subluxation, and cervical canal stenosis. After a detailed neurologic evaluation, we suspected that the patient had an acute BPI.

The patient was prescribed $300 \mathrm{mg}$ of oral gabapentin 3 times a day and a course of oral prednisone beginning at $60 \mathrm{mg}$, with a 10-mg taper on successive days. She also underwent physical therapy for her left arm. Neurologic examination on postoperative day 2 showed $4 / 5$ motor strength of the left arm, with normal biceps and triceps reflexes. She was subsequently discharged on postoperative day 2; continued her oral medications, as well as outpatient physical therapy; and reported no residual neurologic deficits during her postoperative visits 1,3 , and 6 weeks later.

\section{DISCUSSION}

Although BPIs are rare complications, they have been well described in a variety of surgical settings. ${ }^{4-6}$ It is essential to distinguish between BPIs that occur during median sternotomy or spinal surgery and those that occur as a result of patient positioning during surgery. ${ }^{4-6}$ BPIs resulting from laparoscopic or robot-assisted surgery requiring prolonged periods of Trendelenburg positioning remain uncommon, complicating only $0.16 \%$ of all surgical procedures. ${ }^{2}$ Yet, these injuries have significant postoperative morbidity and medicolegal implications. ${ }^{7-10}$ We searched Medline using PubMed for all English-language articles published between 1980 and 2012 with search terms including "brachial plexus injury," "brachial plexus neuropathy," "brachial plexopathy," and "laparoscopy." Although cases associated with postoperative BPIs after laparoscopic general, urologic, 
and gynecologic surgery have previously been reported, we found no cases of BPI after robot-assisted gynecologic surgery. The findings of our literature search are summarized in Table 1.6,11-25

The brachial plexus, comprising nerves $\mathrm{C} 5$ to T1, runs beneath the clavicle and enters the arm medial to the humeral head. ${ }^{1,3}$ Normally, neighboring bones protect the brachial plexus from injury; however, the proximity of the brachial plexus to these mobile neighboring bony structures leaves it vulnerable to injury related to malpositioning. ${ }^{1,3}$ BPIs may occur when the brachial plexus is stretched or compressed. ${ }^{1,3}$ Stretch injuries usually occur when shoulder braces are placed too laterally, especially when the arm is abducted. ${ }^{1,3}$ Two opposing forces stretch the nerves of the brachial plexus: an upward force on the shoulder by the shoulder brace and the downward force of gravity on the patient.1.,3 In contrast, compression injury occurs when shoulder braces are placed too close to the neck with the arm hyperabducted, thereby compressing the brachial plexus against the first rib., 3,7

Injuries to peripheral nerves occur because of a combination of stretch and compression that ultimately causes local ischemia to these nerves. ${ }^{7}$ Several risk factors have been implicated in the development of intraoperative BPIs. ${ }^{1-3,7,8}$ The Trendelenburg position is often used during laparoscopic gynecologic surgery to facilitate intraabdominal exposure; however, shoulder braces used in conjunction with the Trendelenburg position predispose patients to BPIs. In this scenario the force of the shoulder braces on the clavicle and scapula opposes the force of gravity on the humerus, thereby stretching the brachial plexus and leading to a nerve injury. ${ }^{1-3,7,8}$ This risk is particularly exaggerated when the arm is hyperabducted $\left(>90^{\circ}\right)$, the head is laterally flexed to the opposite side, or the abducted arm is sagging. ${ }^{25}$ With increased operating times, patients remain under general anesthesia longer; this increases the risk of a BPI by increasing joint mobility (particularly when muscle relaxants are used) because the neighboring bony structure is more likely to compress or impinge on the brachial plexus. ${ }^{3}$ Patients with extreme body mass index (BMI) values, cervical spine abnormalities, congenital anomalies such as a cervical rib, or preexisting diabetes or other chronic vascular diseases are also more prone to the development of BPIs. ${ }^{1,3,7}$ Moreover, hypothermia, hypovolemia, and hypotension during surgery may contribute to the risk of BPIs developing. ${ }^{1}$ Finally, electrolyte imbalances, vitamin $\mathrm{B}_{12}$ deficiency, alcoholism, and smoking may predispose patients to the development of neuropathies. ${ }^{1}$
Our search for cases of laparoscopic gynecologic surgery complicated by BPIs showed 8 published reports, which highlight several risk factors (Table 1). The Trendelenburg position was used in all cases, with a mean tilt angle of $28.5^{\circ}$ (range, $20^{\circ}-38^{\circ}$ ). Shoulder braces, however, were used in only 6 cases. Five cases reported patient positioning with 1 arm abducted; in 2 additional cases, patients had both arms abducted. The mean operating time was 189.1 minutes (range, 60-360 minutes), and the mean BMI of patients was $32.2 \mathrm{~kg} / \mathrm{m}^{2}$ (range, $19.6-70 \mathrm{~kg} / \mathrm{m}^{2}$ ). Although half these patients were either obese or morbidly obese, the remaining half were at the lower end of normal BMI values.

Consistent with the aforementioned risk factors, our case involved a patient with a low-normal BMI, ${ }^{19}$ who spent approximately 320 minutes in the steep Trendelenburg position. Although the patient's arms were not abducted, shoulder braces were used in this case. We hypothesize that a stretch injury of the brachial plexus developed in our patient because of the combination of shoulder braces, Trendelenburg positioning, and prolonged operating time.

A history of a long laparoscopic or robot-assisted surgery, with reports of pain, numbness, and tingling along one or both arms postoperatively, should always alert a gynecologist to the possibility of a BPI. ${ }^{1}$ Although BPIs can be diagnosed by a combination of clinical history and detailed neurologic findings, magnetic resonance imaging can be used as an adjunct when cervical radiculopathy is suspected. ${ }^{1}$ Although most BPIs are transient and resolve spontaneously, depending on the extent of ischemia to the brachial plexus, BPIs can lead to short- or long-term dysfunction, including tingling, numbness, pain, and loss of function in the upper extremities. ${ }^{1,3,8}$ The mainstay of treatment is physical therapy of the affected side, as well as oral nonsteroidal anti-inflammatory drugs, or antiepileptic agents such as gabapentin or topiramate if the patient has neuropathic pain. ${ }^{1}$ Treatment efficacy is monitored clinically; however, nerve conduction studies and electromyography may be required to evaluate the extent of injury if neurologic symptoms persist for $>4$ weeks. ${ }^{1}$ The diagnosis of BPI in our patient was achieved clinically, and in conjunction with physical therapy and oral analgesics, the patient showed resolution of symptoms within 4 weeks of her initial surgery.

A consistent and standardized approach to patient positioning is integral to any surgical procedure because the patient is subject to intraoperative complications as soon as he or she is placed on the operating table. ${ }^{8}$ Prevention 


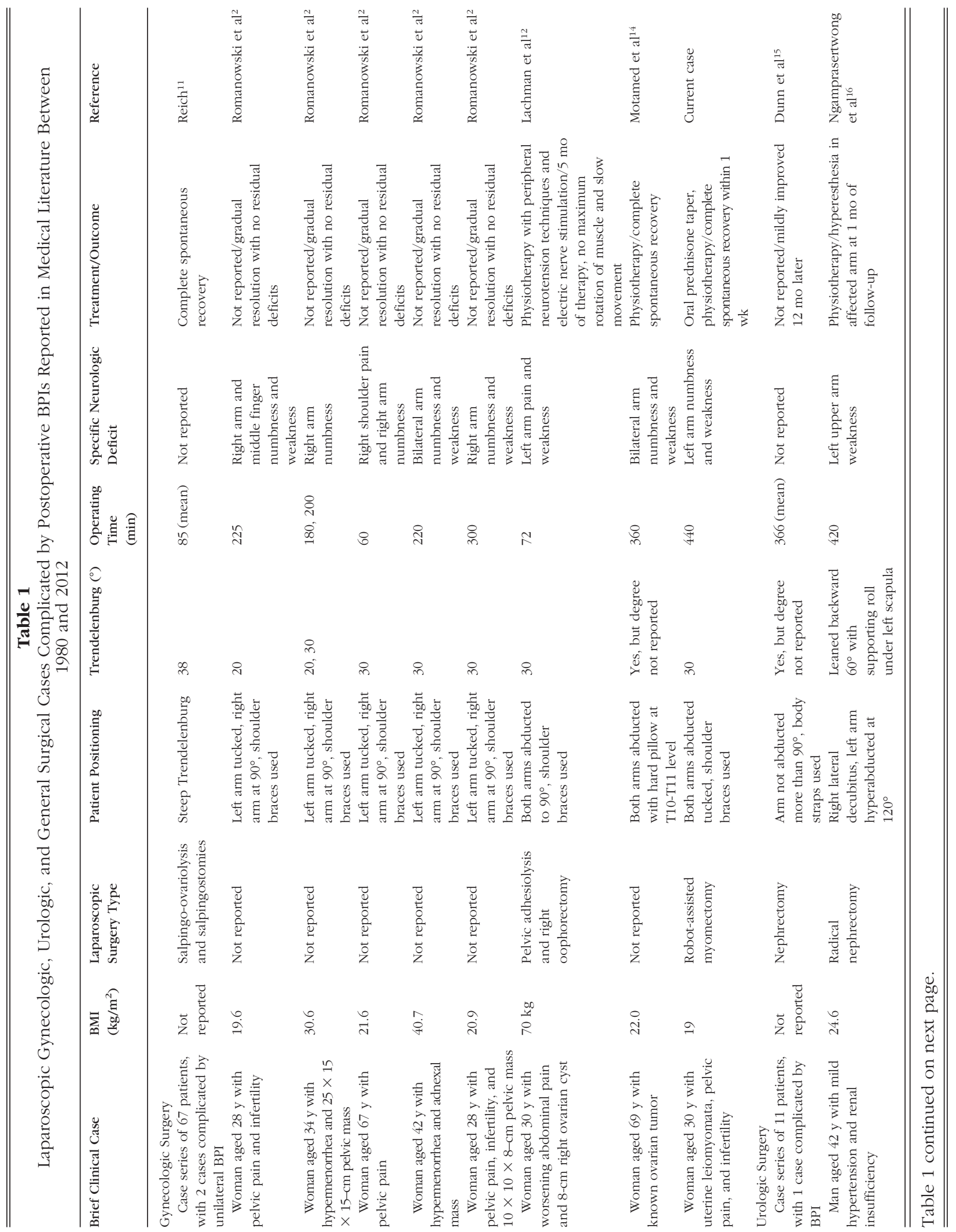




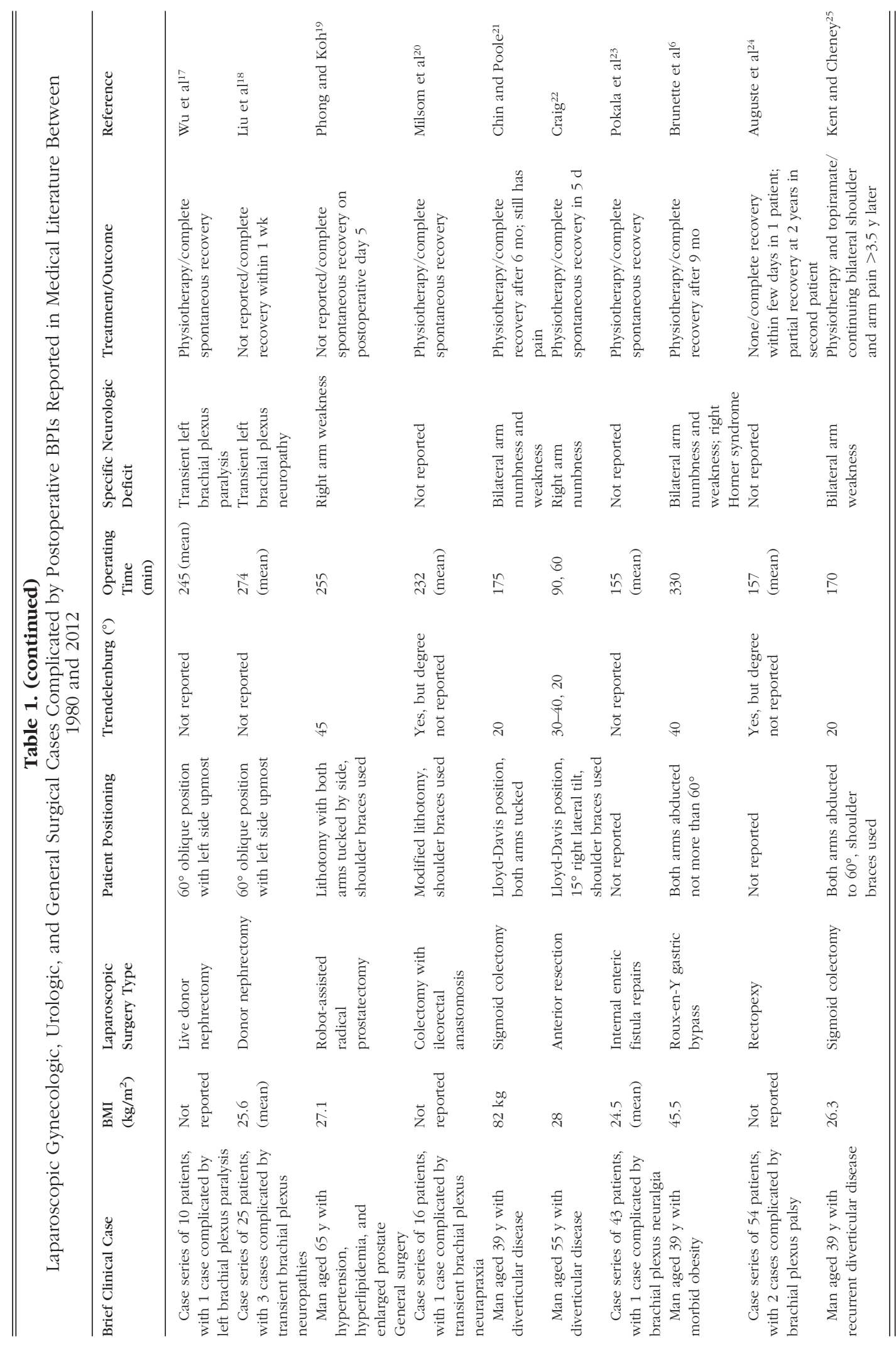


of intraoperative BPIs, particularly during laparoscopic and robot-assisted gynecologic surgery, should take into account the anatomy of the brachial plexus, as well as the aforementioned risk factors that predispose patients to the development of BPIs. ${ }^{1,8}$ First, the time spent in the Trendelenburg position, as well as the tilt angle, should be minimized without compromising surgical exposure. ${ }^{1}$ A recent study by Ghomi et $\mathrm{al}^{13}$ has questioned the utility of steep Trendelenburg positioning in gynecologic surgery, particularly robot-assisted laparoscopic surgery. They used a mean tilt angle of $16.4^{\circ}$ and concluded that robotassisted laparoscopic surgery could be performed effectively without using steep Trendelenburg positioning. They emphasized that a surgeon's skill and experience should dictate the degree of Trendelenburg positioning to achieve surgical exposure, rather than routinely placing all patients in the steep Trendelenburg position. Minimizing the tilt angle would thereby decrease any intraoperative or postoperative respiratory, circulatory, or neurologic complications. Second, use of shoulder braces or restraints should be limited; instead, alternatives such as cross-chest straps, beanbags, gel pads, or egg crate foam mattresses should be used to prevent the patient from slipping cephalad during surgery. ${ }^{1,3,8}$ If shoulder braces must be used, proper placement over the acromioclavicular joint should be ensured. ${ }^{3}$ Third, use of arm boards should be prohibited; the patient's arms should instead be tucked to the side with the hands facing his or her thighs. ${ }^{1}$ Finally, members of the surgical team and operating room staff should remain cognizant of the operating time so that adjustments to the patient's intraoperative position can be made when necessary. ${ }^{1}$

Minimally invasive surgical options, particularly laparoscopic and now robot-assisted laparoscopic surgery, have revolutionized the surgical management of different gynecologic conditions. These surgical procedures often require longer operating times because of instrument preparation and setup as well as physician technique; that is, they have a learning curve. ${ }^{8}$ Because patients inevitably spend longer times in the Trendelenburg position during gynecologic surgeries, intraoperative nerve injuries related to patient positioning are bound to occur. Although nerve injuries such as BPIs are rare complications of surgery, these injuries may be debilitating and can pose medicolegal implications as well. ${ }^{1,3,8}$

Patient positioning in the operating room should be a team effort that optimizes surgical efficacy without compromising patient safety. ${ }^{3,8}$ To achieve this, members of the nursing, surgical, and anesthesia teams must make a thorough preoperative assessment to identify risk factors that make the patient susceptible to injury. During long laparoscopic and robot-assisted gynecologic surgeries, the anesthesiologist should make repeated intraoperative assessments to recognize and address any causes of positioning-related BPIs. Because the patient's arms remain outside the surgeon's field of vision, the anesthesiologist should take precautions regarding the placement and stability of the patient's arms. ${ }^{12}$ Although nerve injuries due to patient positioning may still occur despite adequate preventive measures, understanding proper neuroanatomic relationships, mechanisms of injury, and risk factors that make patients prone to such injuries can help minimize these surgical complications. ${ }^{1,3,8}$

\section{References:}

1. Shveiky D, Aseff JN, Iglesia CB. Brachial plexus injury after laparoscopic and robotic surgery. J Minim Invasive Gynecol. 2010;17:414-420.

2. Romanowski L, Reich H, McGlynn F, Adelson MD, Taylor PJ. Brachial plexus neuropathies after advanced laparoscopic surgery. Fertil Steril. 1993;60:729-732.

3. Bradshaw AD, Advincula AP. Postoperative neuropathy in gynecologic surgery. Obstet Gynecol Clin North Am. 2010;37: 451-459.

4. Uribe JS, Kolla J, Omar H, et al. Brachial plexus injury following spinal surgery. J Neurosurg Spine. 2010;13:552-558.

5. Canbaz S, Turgut N, Halici U, Sunar H, Balci K, Duran E. Brachial plexus injury during open heart surgery-controlled prospective study. Thorac Cardiovasc Surg. 2005;53:295-299.

6. Brunette KE, Hutchinson DO, Ismail H. Bilateral brachial plexopathy following laparoscopic bariatric surgery. Anaesth Intensive Care. 2005;33:812-815.

7. Winfree CJ, Kline DG. Intraoperative positioning nerve injuries. Surg Neurol. 2005;63:5-18.

8. Agostini J, Goasguen N, Mosnier H. Patient positioning in laparoscopic surgery: tricks and tips. J Visc Surg. 2010;147:e227e232.

9. Frederick W, Cheney MD. The American Society of Anesthesiologists closed claims project. Anesthesiology. 1999;91:552556.

10. Cheney FW, Domino KB, Caplan RA, Posner KL. Nerve injury associated with anesthesia: a closed claims analysis. $A n-$ esthesiology. 1999;90:1062-1069.

11. Reich H. Laparoscopic treatment of extensive pelvic adhesions, including hydrosalpinx. J Reprod Med. 1987;32:736-742. 
12. Lachman E, Rosenberg P, Gino G, Levine S, Goldberg S, Borstein $\mathrm{M}$. Axonal damage to the left musculocutaneous nerve of the left biceps muscle during laparoscopic surgery. J Am Assoc Gynecol Laparosc. 2001;8:453-455.

13. Ghomi A, Kramer C, Askari R, Chavan NR, Einarsson JI. Trendelenburg position in gynecologic robotic-assisted surgery. J Minim Invasive Gynecol. 2012;19(4):485-489.

14. Motamed C, Gouy S, Bourgain JL. Bilateral postoperative brachial plexus palsy and longlasting surgery. EurJ Anaesthesiol. 2011;28:608-609.

15. Dunn MD, Portis AJ, Elbahnasy AM, et al. Laparoscopic nephrectomy in patients with end-stage renal disease and autosomal dominant polycystic kidney disease. Am J Kidney Dis. 2000;35:720-725.

16. Ngamprasertwong P, Phupong V, Uerpairojkit K. Brachial plexus injury related to improper positioning during general anesthesia. J Anesth. 2004;18:132-134.

17. Wu CT, Chiang YJ, Liu KL, Chu SH. Laparoscopic donor nephrectomy: new combination of hand-assisted and standard approaches. Transplant Proc. 2004;36:1909-1911.

18. Liu KL, Chiang YJ, Wu CT, Lai WJ, Wang HH, Chu SH. Why we consistently use the left donor kidney in living related transplantation: initial experience of right laparoscopic donor ne- phrectomy and comparison with left nephrectomy. Transplant Proc. 2006;38:1977-1979.

19. Phong SV, Koh LK. Anaesthesia for robotic-assisted radical prostatectomy: considerations for laparoscopy in the Trendelenburg position. Anaesth Intensive Care. 2007;35:281-285.

20. Milsom JW, Ludwig KA, Church JM, Garcia-Ruiz A. Laparoscopic total abdominal colectomy with ileorectal anastomosis for familial adenomatous polyposis. Dis Colon Rectum. 1997;40: 675-678.

21. Chin P, Poole G. Bilateral brachial plexus injury during laparoscopic sigmoid colectomy. ANZ J Surg. 2003;73:86-88.

22. Craig J. Shoulder supports, brachial plexus injury and headdown tilt. Anaesthesia. 2004;59:196.

23. Pokala N, Delaney CP, Brady KM, Senagore AJ. Elective laparoscopic surgery for benign internal enteric fistulas: a review of 43 cases. Surg Endosc. 2005;19:222-225.

24. Auguste T, Dubreuil A, Bost R, Bonaz B, Faucheron JL. Technical and functional results after laparoscopic rectopexy to the promontory for complete rectal prolapse. Prospective study in 54 consecutive patients. Gastroenterol Clin Biol. 2006;30:659-663.

25. Kent CD, Cheney FW. A case of bilateral brachial plexus palsy due to shoulder braces. J Clin Anesth. 2007;19:482-484. 